\title{
Development of SiN-coated Carbon Membranes for Environmental-cell Window
}

\author{
T. Kawasaki ${ }^{1,3}$, Z. Cui $^{1}$, N. Imaeda ${ }^{1}$ and T. Tanji ${ }^{2,3}$ \\ 1 Dept. Electrical Eng., Nagoya Univ., Furo-cho, Chikusa-ku, Nagoya, 464-8603, Japan \\ ${ }^{2}$ EcoTopia Science Inst., Nagoya Univ., Furo-cho, Chikusa-ku, Nagoya, 464-8603, Japan \\ 3 Global Research Center for Environment and Energy based on Nanomaterials Science (GREEN), \\ 1-2-1 Sengen, Tsukuba, 305-0047, Japan
}

In-situ controlled-environment TEM (ETEM) is a powerful technique to study nature of materials under the conditions in which they are formed or applied. In cases of actual materials such as catalysts, fuel cells, biological molecules, etc., it is essential to obtain their practical information, not that in laboratory. The authors has been also developed a closed-type environmental-cell (E-cell) system and applied to observations of Au nanoparticulate catalysts [1]. In the above system, window material is one of the most important components because it must seal gases inside the E-cell and its thickness must be thin enough to suppress electron scattering in the membranes. Amorphous carbon is suitable to this purpose, because light and conductive element has less influence on illuminated electron beam and thin film ( $<10 \mathrm{~nm}$ thick) enabling to withstand atmospheric pressure difference can be obtained [2]. However, carbon films have disadvantage to be easily damaged by the electron beam irradiation under oxygen-containing gas environment. Since silicon nitride $(\mathrm{SiN})$ is relatively stable for the electron beam, it has been applied as another window material. Therefore, hybridization of the carbon and the SiN was expected to show high performance such as less deterioration of imaging resolution, high toughness for pressure difference, and high stability for electron beam irradiation. In the present paper, we report about thickness of coated $\mathrm{SiN}$ and toughness against electron beam irradiation.

Figure 1 shows a schematic illustration of our developed environmental-cell. The membrane coated by $\mathrm{SiN}$ is supported on a dedicated $\mathrm{Cu}$ grid. Specimens are set between two membranes separated by spacers. The carbon film of $8 \mathrm{~nm}$ thickness was developed by the dedicated vacuum deposition technique [2]. The amorphous $\mathrm{SiN}$ was deposited on the carbon with controlling its thickness up to $2 \mathrm{~nm}$ to an accuracy of $\sim 0.1 \mathrm{~nm}$ by the pulsed laser deposition (PLD) method. In order to evaluate toughness of these membranes against electron beam irradiation, TEM images of the membranes were taken at a few or several minutes intervals during in-situ experiments under gas environment (CO content of $1 \%$ in artificial air at $1000 \mathrm{~Pa}$ ). When thickness of the coated $\mathrm{SiN}$ was $1.0 \mathrm{~nm}$, the membrane was broken after 13 minutes from the beginning of the irradiation, as shown in Figure 2(a). In contrast, 1.5nm-thick SiNcoated membrane, (b), suffered almost no damage for more than 20 minutes. The X-ray photoelectron spectroscopy (XPS) analysis was done to understand the cause of this difference. Figure 3 shows XPS spectra of the carbon membranes coated by $\mathrm{SiN}$ of $1.0 \mathrm{~nm}((\mathrm{a}),(\mathrm{c}))$ and $1.5 \mathrm{~nm}$ ((b), (d)). The upper and lower side spectra correspond to N1s peak and Si2p peak, respectively. Here, content of Si-N bond (red color spectra) in the $1.5 \mathrm{~nm}$-thick SiN-coated membranes was three times bigger than that in the $1.0 \mathrm{~nm}$ thickness. On the other hand, undesired Si-O bonds and N-C bonds were decreased. These results represented that main composition of the deposited film was not $\mathrm{SiN}$ but $\mathrm{SiO}$ and $\mathrm{CN}$ at the beginning of the film formation. When thickness of coating layer became more than $1.5 \mathrm{~nm}$, composition of the layer changed to the SiN. Therefore, we adopted the membranes coated by the $\mathrm{SiN}$ of more than $1.5 \mathrm{~nm}$ thickness as the environmental-cell window material.

\section{References}

[1] T. Kawasaki et al., Proc. M\&M2011. (2011) 465.

[2] K. Ueda et al., Surf. Interface Anal. 40, 1725 (2008). 


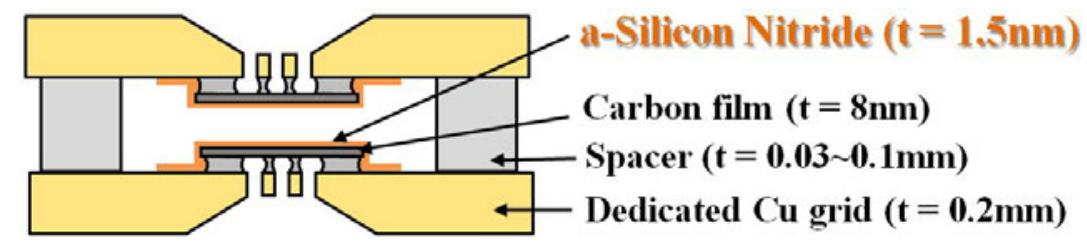

Figure 1. Schematic illustration of our windowed type environmental-cell.

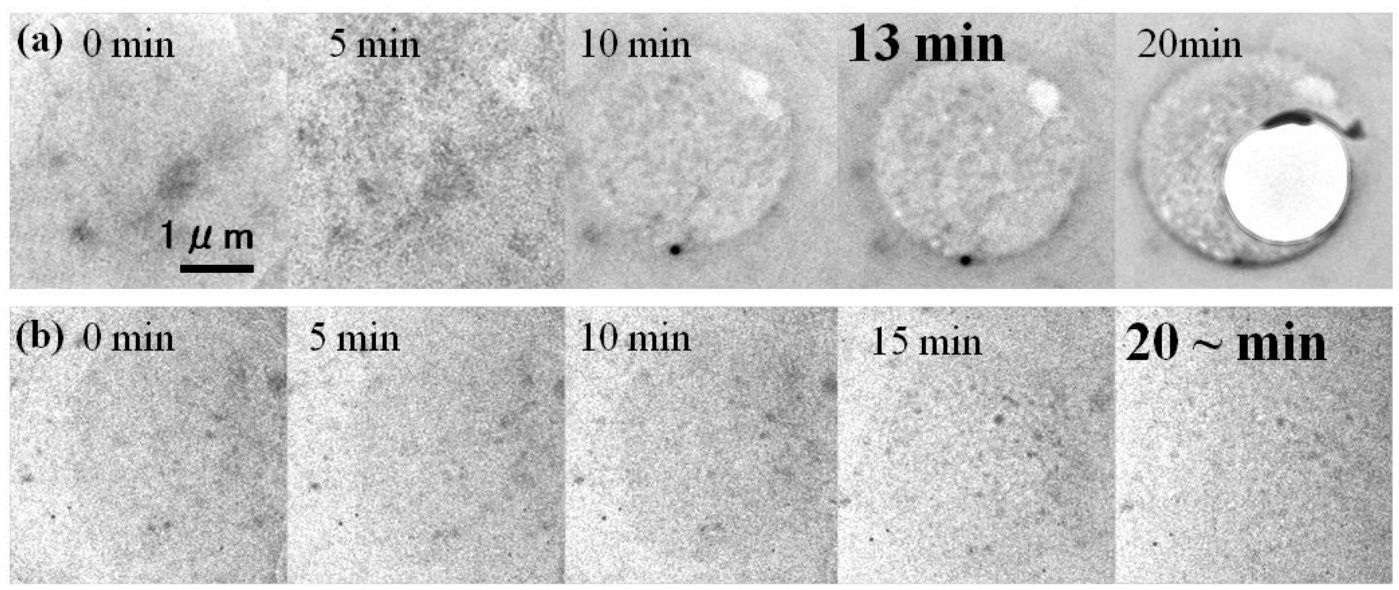

Figure 2. TEM images of the membranes after in-situ observation under gas environment (Air + CO; 1000Pa). (a) carbon $(8 \mathrm{~nm})+\mathrm{SiN}(1.0 \mathrm{~nm})$, (b) carbon $(8 \mathrm{~nm})+\operatorname{SiN}(1.5 \mathrm{~nm})$.
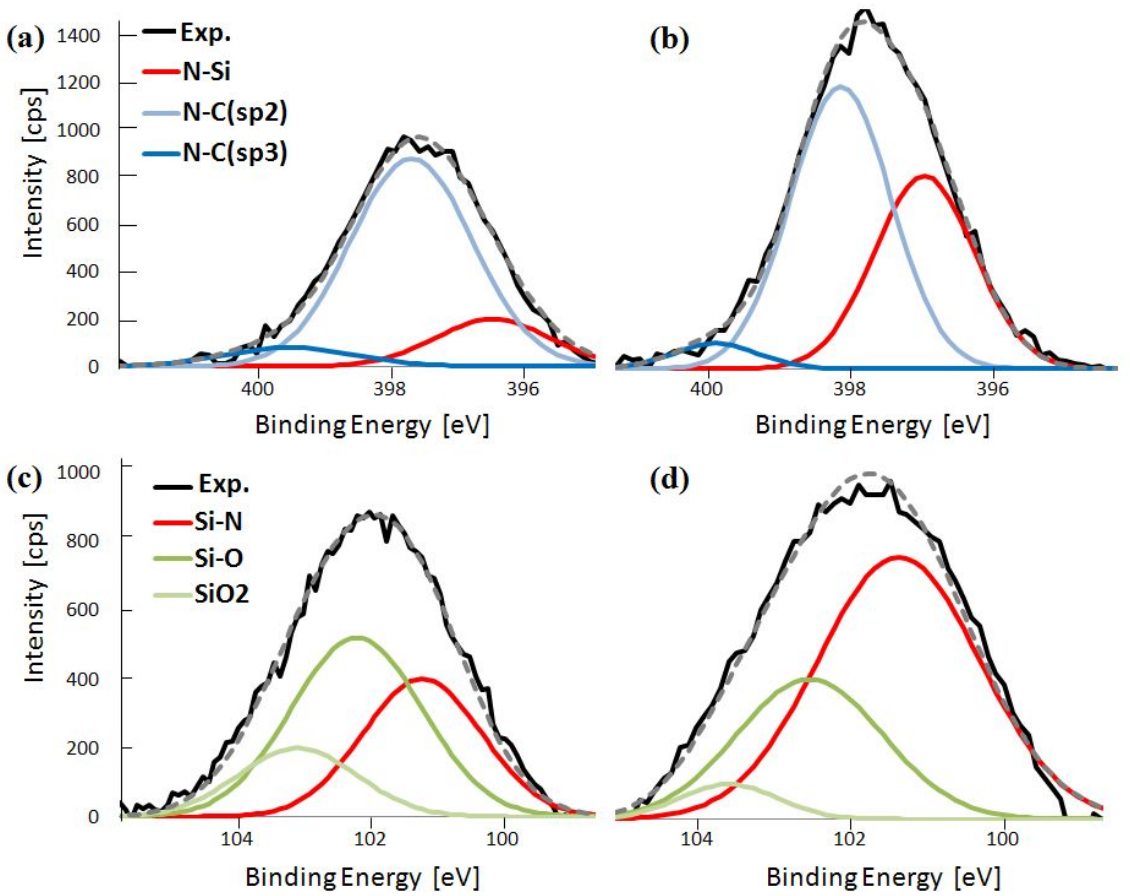

Figure 3. XPS spectra of the carbon membranes coated by SiN of (a), (c) $1.0 \mathrm{~nm}$ and (b), (d) $1.5 \mathrm{~nm}$ thickness. The upper and lower side spectra correspond to N1s peak and Si2p peak, respectively. 